\title{
A Game Theoretic Approach to Demand Side Management in Smart Grid with Multiple Energy Sources and Storage
}

\author{
Aritra Kumar Lahiri, Ashwin Vasani, Sumanth Kulkarni, Nishant Rawat \\ School of Computing, Informatics, \\ Decision Systems Engineering, \\ Arizona State University \\ Tempe, USA
}

\begin{abstract}
A smart grid is an advancement in electrical grid which includes a variety of operational and energy measures. To utilize energy distribution in an efficient manner, demand side management has become the fore-runner. In our research paper, we use game theory as a tool to model our system as a Stackelberg game. We make use of different energy sources like solar energy, energy from battery and energy from the provider to run appliances of a subscriber. We consider the scenario where the subscriber can give excess energy that is generated, back to the grid, thereby reducing the load on the grid during peak hours. We design a pricing scheme to calculate the utilities of the subscriber and the provider and show how our model maximizes the utility of the entire system, thereby showing the existence of a Nash equilibrium.
\end{abstract}

Keywords-Demand side management; utility; smart grid; solar; battery; energy provider; fairness; proportional division; utilitarian division; Nash equilibrium

\section{INTRODUCTION}

Traditional grids have been around for more than a 100 years. They were developed to meet the energy needs of the 19 th century. Demands of the 21 st century are ever changing and despite reasonable patchwork, traditional grids are not capable to adapt to that change any more. This brings smart grid into the picture. Smart grid is an electrical grid which allows two-way communication between providers and subscribers. It makes use of information technology to quickly adapt to the changing needs of the subscribers. Smart grid provides digitization of communications by aligning the providers and subscribers in a much more effective way. One of the main emerging areas in the smart grid research is Demand Side Management (DSM) [1], [2].

\section{A. Demand Side Management as a Framework}

Demand side management [1] can be characterized as a set of interconnected and flexible programs that facilitates efficient usage of electric power. It helps in controlling electric power consumption by shifting load accordingly and at the same time making a fair allocation of resources among subscribers. This also ensures that the subscribers are provided incentive when they reduce their consumption during peak hours to the hours when the smart grid is less loaded. Demand side management techniques provides smart pricing schemes that can ensure load shifting by implementing various scheduling schemes for appliances and also managing interactions at social (service level agreement between energy provider and subscribers) as well as technical (between smart meters and energy providers) interaction [3], [4]. The nature of demand side management and smart grid suggests the use of Game Theory as an analytical tool.

\section{B. Game Theory as a Tool}

Game theory is a framework [1] which makes use of mathematical rules and relations to study complex interactions between players. This is the primary reason why game theory can be used in developing an optimal solution for cases of cooperation or conflict with players acting in their best interest. Due to involvement of fair allocation of power and pricing schemes in demand side management, game theory can provide a natural mechanism for developing them.

\section{Multiple Sources of Energy}

We have also explored the possibility of multiple energy sources in this paper and considered solar energy to be an energy source. Solar energy is a form of renewable energy resource, which is cheap and easily available and can be utilized as an alternate way to obtain energy by the subscribers. We present our research in demand side management using storage devices where we are considering low cost efficient energy storing device like battery for charging during off peak hours and discharging during peak hours to reduce the load on the grid as well as give incentives to the subscribers. The consideration of battery is in tune with the fact that weather plays an effective part in obtaining energy from solar resource, hence it ensures that subscribers have sufficient amount of energy to run their basic appliances during hours of high payload. Finally we propose a model where the subscribers are strategically choosing their way of using the storage devices and when to buy energy from the providers.

\section{RELATED WORK AND MOTIVATION}

One of the important papers [1] in this direction is authored by E. Salfati and R. Rabinovici. The research direction of that paper focuses primarily on the single energy provider system with multiple subscribers where the subscribers compete for resources from the energy provider. 
Usually, if all the subscribers are given access to get energy for all their appliances, there is a possibility that the energy demand would exceed the total energy which can be supplied by the grid. Hence we utilize game theory in demand side management to manage the shared resource in a fair manner such that it does not disturb the subscriber interest, plus ensuring optimal usage of the grid.

Our paper focuses on the usage of multiple energy sources. The rise of alternate energy sources, specifically solar energy which an independent subscriber can harness on his own has made subscribers self-sufficient to an extent. We aim to consider a flexible system where subscribers can generate their own power and utilize it, while at the same time having the option to consume power provided by external energy provider. We believe this system increases the subscriber satisfaction since it decreases the cost of energy that a subscriber has to pay for, in case of a single energy provider. But with solar energy, we run into a few roadblocks. Changes in weather make solar energy inconsistent. It cannot be relied on a hundred percent. An effective strategy here would be to use a low cost efficient energy storage device which could be used to harness the solar energy and provide energy to the subscribers when there is little to no solar energy availability. Having multiple sources of energy would be beneficial for the subscriber as well as the energy provider. From the subscribers' perspective, the cost which they have to pay to the energy provider would be lessened and from the energy providers' perspective, the overall load on the grid would be reduced while satisfying the subscribers demand.

\section{PROBLEM STATEMENT}

Traditional smart grids face an immense amount of load during peak hours because of the high energy demand of the subscribers. This is detrimental to both the players because the subscribers need to pay a lot more money during peak hours and the energy provider is not able to meet the demands of all the subscribers, thereby reducing the utility of both players, which in turn reduces the utility of the entire system.

We aim to present a demand side management system that enables subscribers to be self-sufficient. We have to come up with a solution which enables us to use solar energy to help the subscribers increase their utility by generating more energy. But since solar energy is an unreliable source of energy, we also have to harness the excess energy from solar and store it in a low cost energy efficient device - battery, which can be used when solar energy is inconsistent. This would make the subscriber self-sufficient to a large extent. This demand side management system will work to keep the utilization of energy from the grid to as less as possible during peak hours. We aim to do this by suggesting a scheduling scheme for the different energy sources that makes a utilityefficient decision while choosing the correct energy source to employ for the subscriber.

Another important aspect which needs to be addressed is the giving back of excess energy generated by the subscriber to the grid during the peak hours. To effectively do this, we have to come up with a pricing scheme which we will develop in order for users to increase their utility. The pricing scheme has to take into consideration, the amount of energy being taken by the subscribers and the amount of energy being given back to the provider. We have to devise a method to calculate how much the subscribers will get paid based on their peak time assistance. So the challenge is to ensure a synergistic relationship among the provider and subscriber, such that the combined utility of our system is maximized.

\section{OUR RESEARCH GOAL}

Our path of research focuses on a demand side management approach that is concerned with the cumulative load of all the subscribers. Hence we formulate a static noncooperative game with $\mathrm{N}$ subscribers that devise a scheduling algorithm for direct load shifting of appliances based on the Asynchronous Consumption Mode (ACM) mentioned by authors in [1]. ACM is characterized by Quality of Service Metrics (QoS) classified according to subscriber characterization of appliances. Following is a brief overview of these metrics:

1) Constant Consumption Rate (CCR): This level of service includes appliances that must be operated whenever their demand is generated, for e.g. refrigerators and room lights.

2) Available Consumption Rate (ACR): Their priority level of service is just next to CCR and can endure a certain period of delay before they need to be operated after their demand, for e.g. Electric kettle and boiler.

3) Unspecified Consumption Rate (UCR): They have the least priority level and can be operated when there is an excess energy capacity that can be used without hampering the working of appliances with CCR, for e.g. under-floor heating and outdoor lighting.

The objective is to minimize the overall energy consumption from the energy provider and effectively reduce the charges incurred by the subscribers. The non-cooperative game is experimentally shown to have existence of Nash equilibrium at a global optimal point. As mentioned earlier, we have considered further, that along with non-cooperative game our model will be based on multiple energy sources. We have considered energy storage as an essential criterion, since it has a huge potential in impacting the demand side management. For example, it can be assumed that a user might opt to store energy during hours when energy load is very low, so that it can utilize them during peak hours rather than obtaining them from power stations thereby maximizing their own utility and the utility of the energy providers [3]. Traditional grid systems faced issues related to voltage fluctuations and harmonic distortions while integrating small scale renewable energy sources due to lack of synchronization. But introduction of smart grids prevents these outages and provides multiple options of renewable resources to supply energy to the grid through distributed power generation and storage. Solar energy is one such form of renewable source that we are considering in our research in this paper. Usage of solar energy requires an initial installation cost, but nevertheless is a cheap and easily available source of energy for the subscribers. However, since weather plays an important factor in the generation of solar power, hence it is practical for the subscribers to use low cost efficient devices 
like battery to store energy. Using battery will ensure that subscribers can store energy for much longer periods of time and discharge energy from them during peak periods and charging them during low energy demand periods and also from solar energy resource whenever it is available. However, without a proper scheduling algorithm for charging and discharging of storage devices, there might be scenario where all storage devices wanting to charge energy simultaneously leading to a higher peak demand [4], [5]. Thus our primary focus is to devise a non-cooperative game model to address the issue. Hence we have proposed a distributed algorithm that supports all the fairness criteria [1] and helps in maximizing social utility of the system. We consider the four main types of fairness criteria while proposing the algorithm such as:

1) Proportional Division: This criteria ensures every player gets his due share according to his own valuation of the resource.

2) Envy-free Division: This criteria ensures that each player gets a valuation which is at least equal to all other players share of resource.

3) Equitable Division: This criteria ensures each player obtains the exact same valuation of the resource.

4) 4. Utilitarian Division: This fairness criteria ensures that the sum of the individual utilities of all the players is maximized.

Here each subscriber can independently strategize for his energy consumption scheduling based on the QoS metrics and also schedule his charging and discharging of storage device like battery. We have experimentally calculated and verified that this model can lower the total energy cost of the entire system by reducing the peak-to-average ratio and maximization of utilities for each of the subscribers.

Along with this, we have also formulated a Stackelberg game for our model as well as implementing a pricing scheme between the energy providers and subscribers. The subscribers and providers are both players of this game and pricing scheme is determined by the providers. The subscribers will aim to buy extra energy before the typical peak hours and store them in their storage devices while trying to sell it back to the providers during peak hours to minimize their cost. The energy providers on the other hand adjust the pricing scheme accordingly once it sees many subscribers are trying to sell back energy during peak hours and it exceeds the consumption rate at that point of time. This scenario can be effectively modelled as a Stackelberg game [4], keeping in mind that the energy providers will try to maximize their profit and the subscribers will aim to reduce their cost of electricity, thereby maximizing the total utility of the system.

\section{DEMAND Side MANAGEMENT ModEL}

\section{A. System Model}

Our system model includes one energy provider providing energy to $\mathrm{N}$ subscribers. Fig. 1 gives a complete overview on how energy providers and subscribers are connected. We assume that every subscriber has an energy consumption controller device (smart meter) which can efficiently allocate energy to the subscriber as well as keep a history on the energy consumption of an individual subscriber. The uniqueness of our model is we utilize multiple energy sources, in specific, two other energy sources - solar energy and a battery. The subscribers can use the energy generated by the solar panel and use it for themselves (during peak and offpeak hours). A battery is an energy storage device and energy providing device in our model since solar is not always reliable and the charge from battery can be utilized to power the appliances for a subscriber. Another uniqueness of our model is that the subscribers can give back excess energy to the energy provider whenever available, thus generating incentives for the subscriber as well as the energy provider.

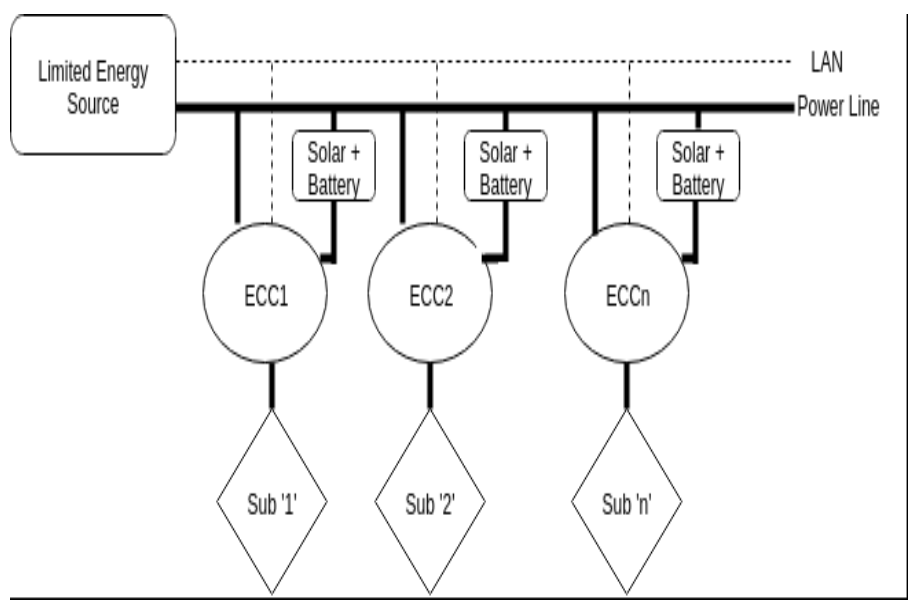

Fig. 1. System Model.

All in all, our model supports energy from three different sources - energy provider, solar energy and battery and the possibility of giving back energy to the grid.

Let $N$ denote the number of subscribers in our model. For every subscriber $n \in N$, we define a parameter called consumption level demand $C_{n}^{d}[k W]$ which is the energy consumed by a particular subscriber. We define our time slot vector $t \in T$ to contain different time slots. $T$ can be any number of divisions for a 24 hour period. The reason for this division is to effectively calculate the subscriber demand for a particular time slot and to calculate the utilities of the subscribers based on whether a particular time slot falls under a peak hour time slot or an off peak hour time slot.

\section{B. Control Loop Description}

The control loop for our model as seen in Fig. 2 is unique since it uses the fair division allocation as seen in [1] as well as considers the usage of solar, battery and energy from the provider. The energy obtained from solar panels is used by the subscriber until it lasts then battery is used. Only if both the solar and battery charge fail to give a certain amount of power which is decided by the subscriber demand algorithm, then energy from the provider is used. Fig. 3 represents the energy supply design. This approach effectively calculates the utilities of the provider and the subscribers by maximizing potential for both the players in our game. The subscribers only pay for the energy they consume depending on the pricing model set by the leader of the game, the energy provider. As discussed in the previous section, the subscribers can also give back 
excess amount of energy generated by them to the energy provider thus receiving incentives.

\section{Game Formulation}

The primary objective of our research is to maximize the utility of the subscribers and the provider, thereby maximizing the utility of the entire system. We model our idea as a Stackelberg game, where the provider is the leader and the subscribers are the followers. The leader has the authority on the pricing model and the constant factor $\delta$ which we will discuss in the upcoming sections. The overall incentives for the subscriber is calculated based on the parameters of amount of energy they are generating, amount of energy they are utilizing, amount of energy they are giving back to the grid.

Provider utility is calculated based on the amount of energy being saved during peak hours. This is discussed in detail in the subsequent sections.

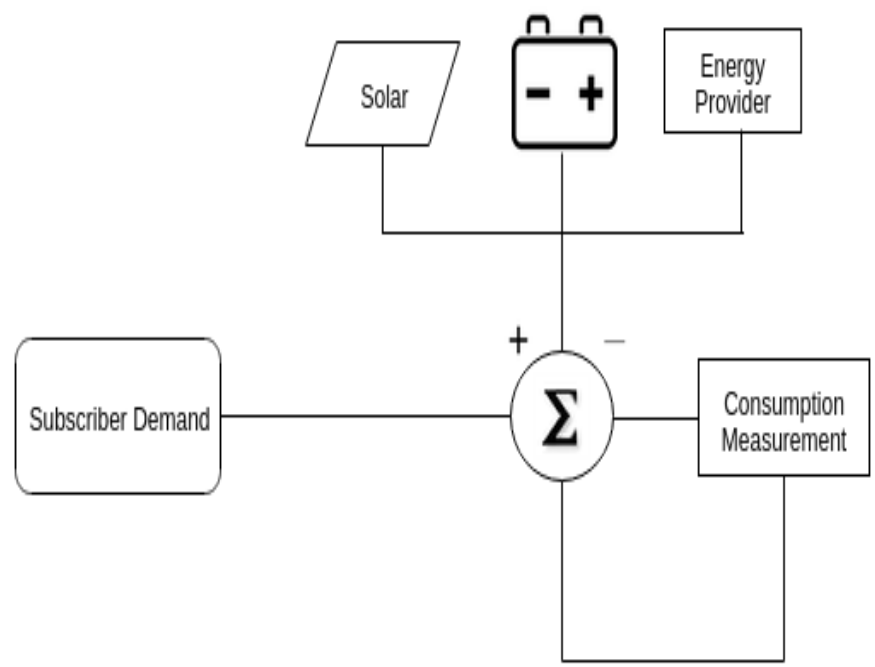

Fig. 2. Control loop description.

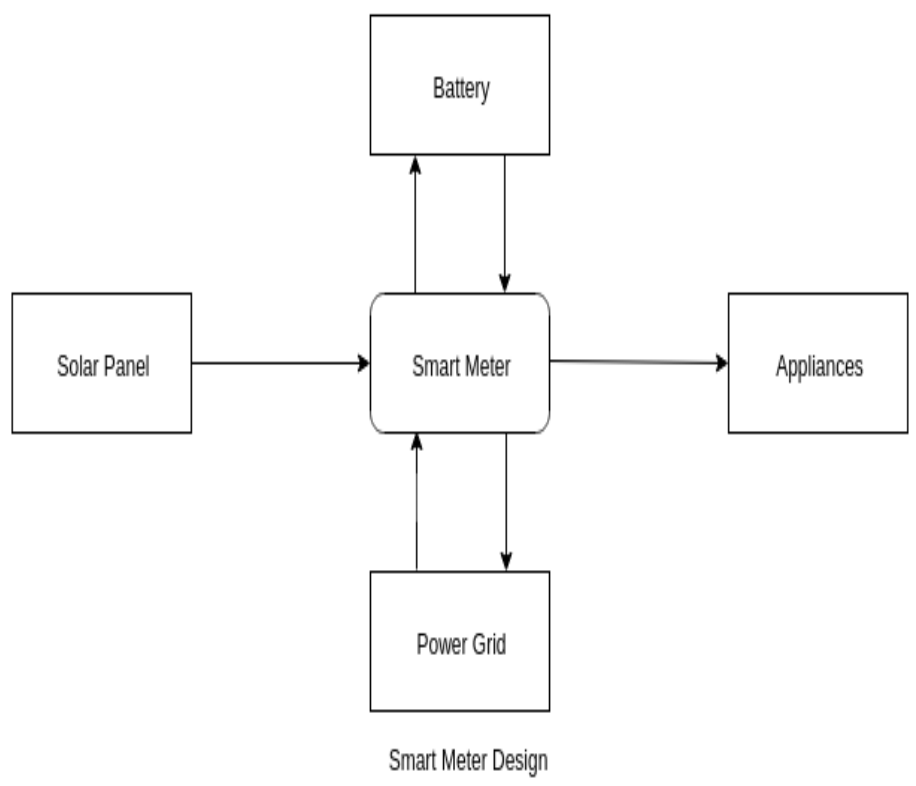

Fig. 3. Energy supply design.

\section{Subscriber Demand Algorithm}

The subscriber demand algorithm determines the amount of energy needed for every subscriber during the peak and offpeak hour time slots. Our analysis has shown that the peak hours are between $12 \mathrm{pm}-7 \mathrm{pm}$ and off-peak hours are between $7 p m-12 p m$ with a majority of energy providers like APS, SRP etc. From [1], we know about the asynchronous consumption mode, which is categorized into three quality of service metrics $Q=1,2,3$ discussed in section 4 , where $j \in Q$, we calculate the energy demand for all the available metrics for every subscriber $k \in N$ depending on the available capacity $C$ of the grid and consumption level demand $C_{n}^{d}[k W]$ of every subscribers in the time slot $T$. Fig. 4(a) to (d) describes the algorithm functionality diagrammatically.

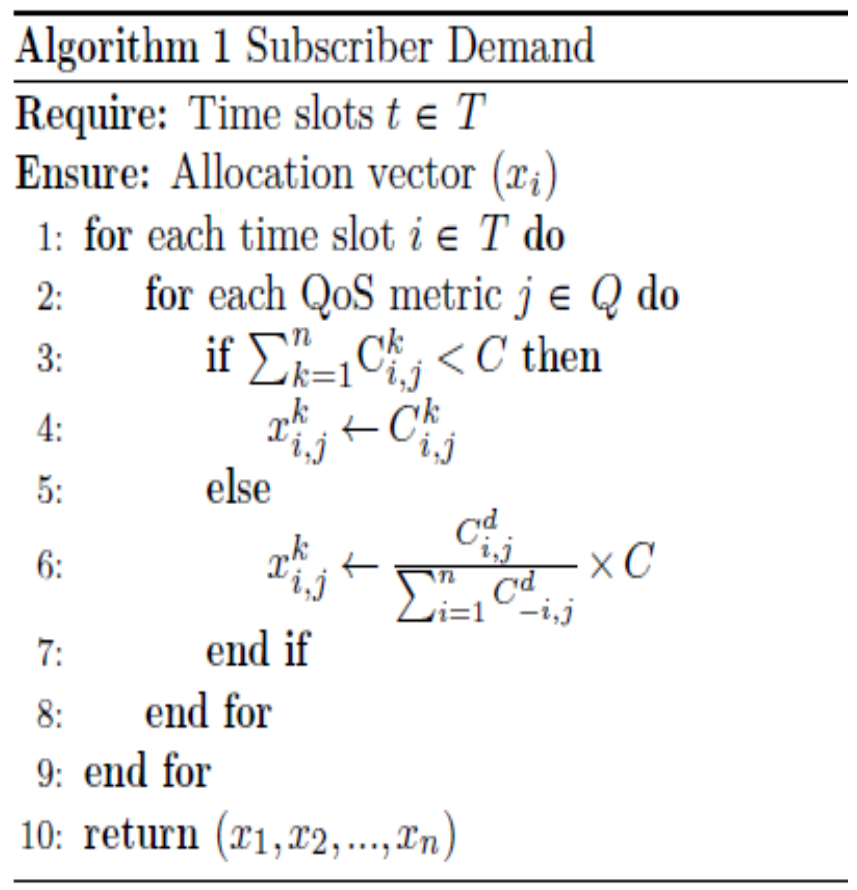

\section{E. Energy Usage Algorithm}

In this section, we describe the algorithm which is used to calculate the total energy usage of a particular subscriber $j \in N$ in time slots $i \in T$ as discussed before. This algorithm considers the amount of solar energy generated along with the battery capacity. If both the primary and secondary energy sources do not satisfy the subscriber demand for different time slots obtained from the subscriber demand algorithm, then energy is obtained from the provider and a cost is paid for it. We define an energy usage vector as $A_{j}^{i}$, solar energy as $s_{j}^{i}$, , battery capacity as $B$, battery charge as $b j$, the charging rate of battery as $c_{r}$, discharging rate of battery as $d_{r}$ and the number of hours the battery charges as $h_{r}$. On the other hand if solar energy is abundant, then we charge the battery as well as give back to the provider for an incentive. Our algorithm takes care of all possible scenarios from the subscriber perspective. The algorithm is as follows: 


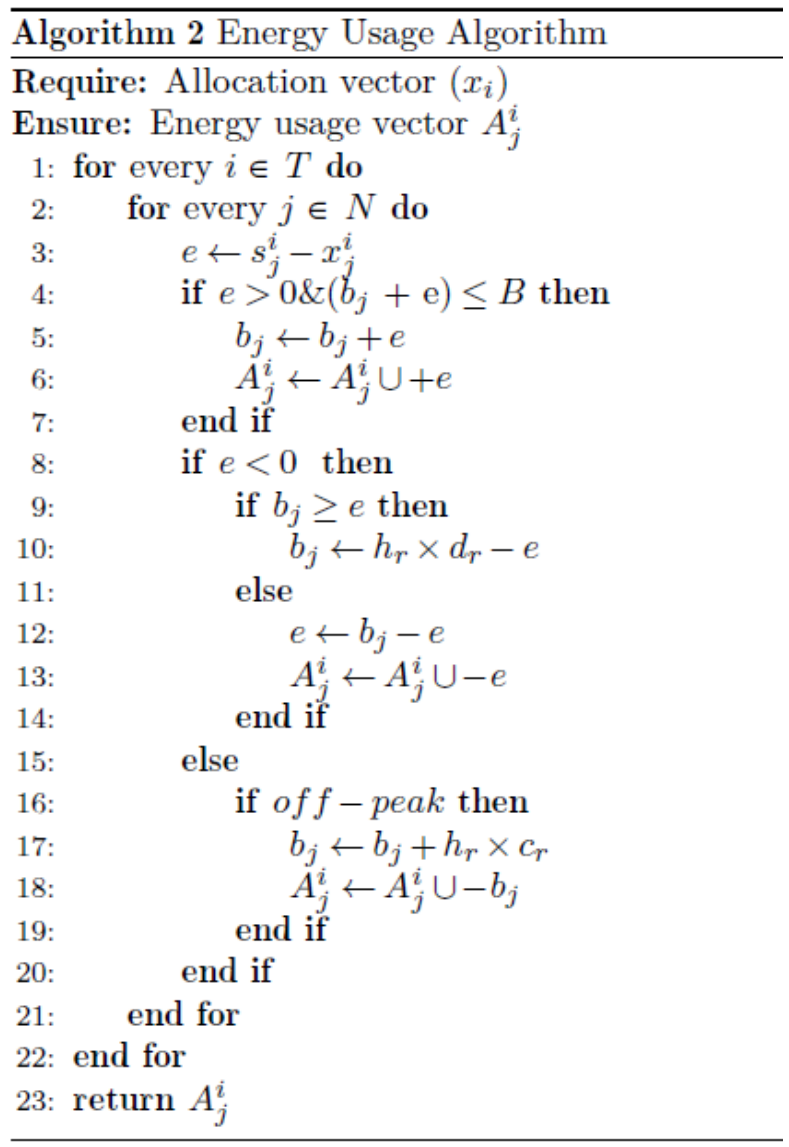

\section{F. Utilitarian Algorithm}

The last algorithm in our model, which we have come up with takes into account the excess energy produced by the subscriber which is given back to the provider and also the energy obtained by the subscriber from the provider. It gets the energy usage vector $A_{j}^{i}$ from Algorithm 2. This algorithm calculates the utilities of the subscribers and the providers. Here, we also introduce a variable $\delta$, which is the basis of our novel pricing model. The utilities of subscribers $u_{j}$ is calculated by taking the difference of the energy supplied to the provider obtained from the energy usage vector $A_{j}^{i}$ and the energy obtained from the provider.

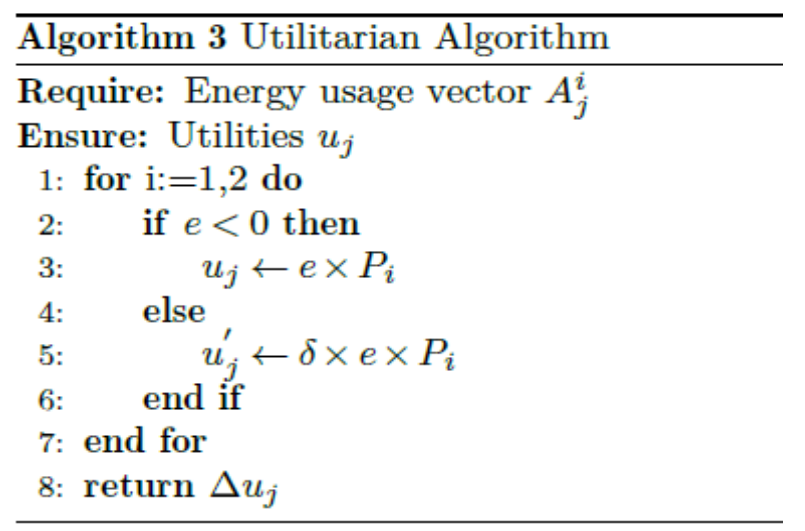

\section{1) Pricing Scheme}

In our approach, we surveyed the pricing schemes of multiple energy providers and we reached a consensus that effective pricing schemes are categorized based on two major grouping of times - peak and off-peak hours. $\delta$ is a variable with value in $[0,1]$. As mentioned before, the leader of the game (provider) sets this value. This value is utilized when the subscribers give energy to the energy provider. If the energy provider needs the energy, he will set the value of $\delta$ to be $>=0$ or $<=1$. Otherwise if the provider does not need the energy from the subscribers, then he could set the value of $\delta$ to be 0 .

The utility of subscribers can be obtained by $u_{j}=u_{j}-u_{j}^{0}$. This is the amount of money that the subscribers save or get from the energy provider.

On the other hand, the provider utility $u_{p}$ can be obtained by the energy being used by the subscribers and the energy received from the subscribers.

The utility of provider can be obtained by $u_{p}=u_{j}^{0}$ since this corresponds to the energy received from the subscribers and he also make a monetary gain with the amount of power consumed by the subscribers specified in $u_{j}$. Owing to these conditions, we see that the utilities of the subscribers and the provider are maximized from our algorithm.

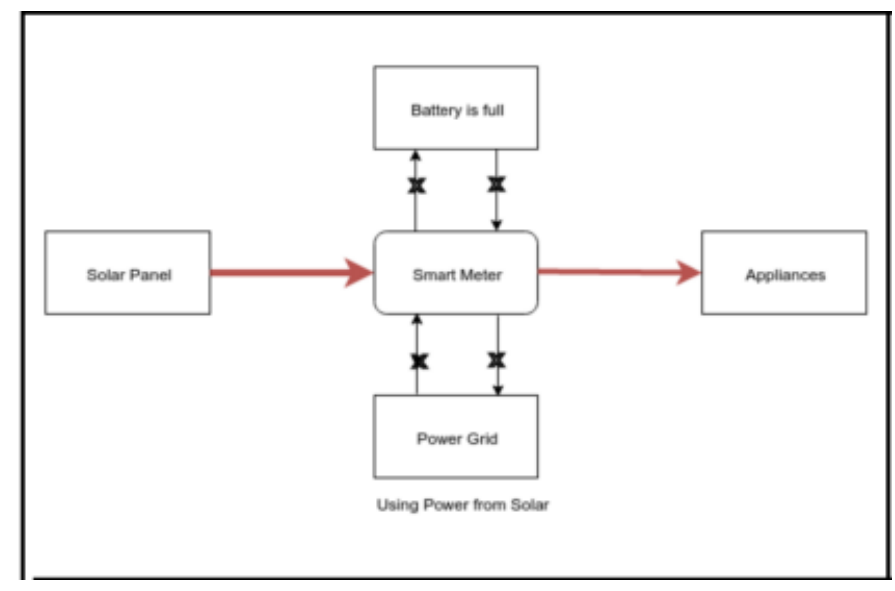

(a) Smart meter actions using power from solar.

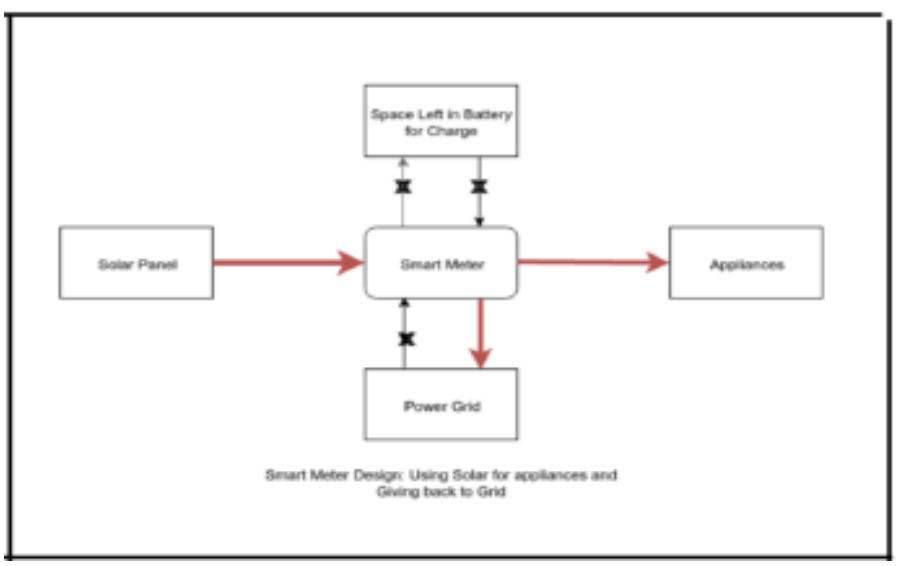

(b) Smart meter action using solar for appliances and getting back to grid. 


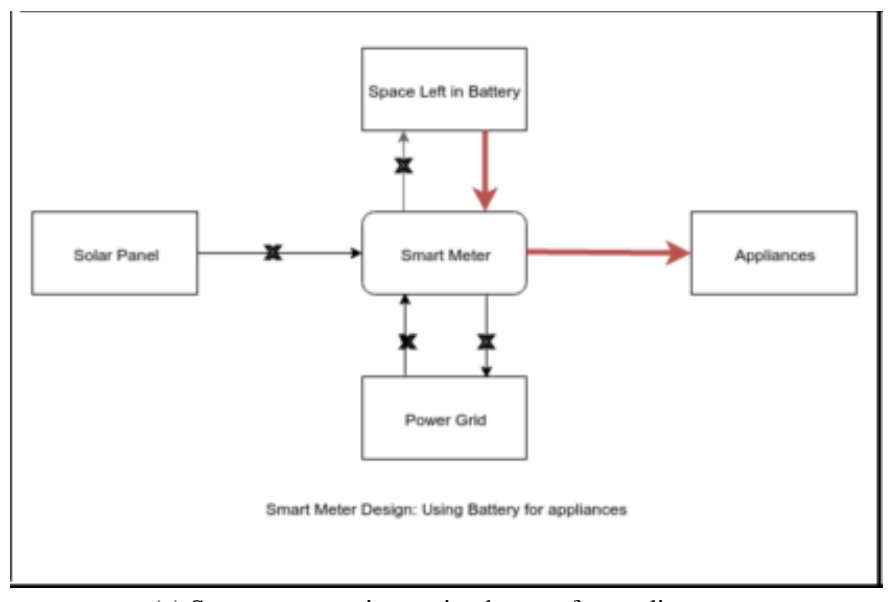

(c) Smart meter actions using battery for appliances.

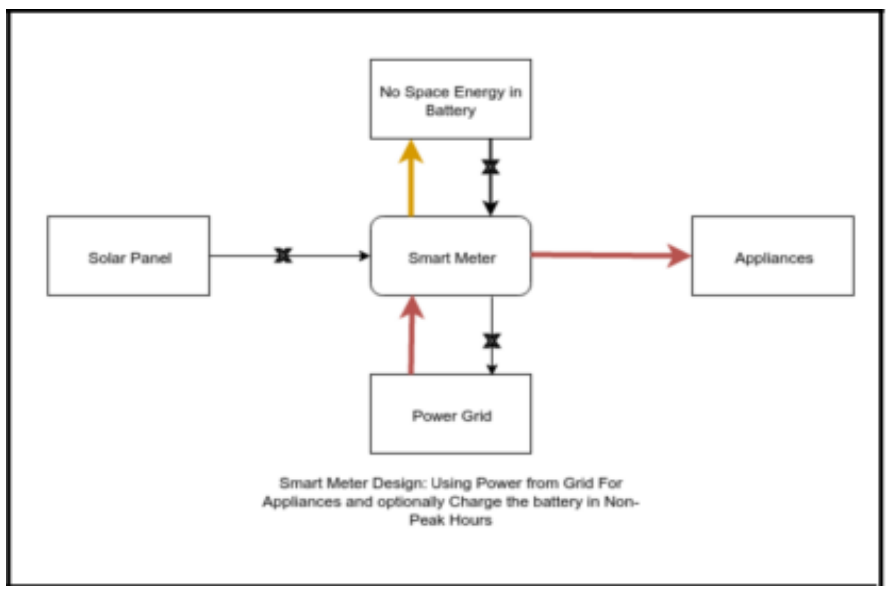

(d) Smart meter actions using power from grid and optionally charging battery in non-peak hours.

Fig. $4 . \quad$ (a)-(d) Smart meter actions.

\section{G. Existence of Nash Equilibrium}

From the utilitarian algorithm, we see that the subscribers make a monetary profit when they give energy back to the provider, thus enhancing their utility. They save up money by using multiple sources of energy as well as receive incentive for giving energy to the grid. The provider profit is also maximized because the subscribers manage the peak hour load as show in Algorithm 2 alongside having a monetary gain from the power distributed during the off peak hours. Since the utilities of both the provider and the subscribers are at maximum, we can effectively say that utilitarian algorithm gives us a Nash Equilibrium. Our algorithm also satisfies the fairness criteria mentioned in Section 4.

\section{Performance EVAluation}

For simulation, we have taken values considering a real life scenario. Our designed algorithm will work on smart meter which has a capacity to control source and destination using digital relay circuits. In our system, we assume that the battery is a two way source, which means the battery can be charged or discharged. In discharging phase, the energy will not flow back to the grid. The grid also has two way capacity. Energy can be given to the grid or it can take back. Solar is a single point source which can generate the energy and appliance can use the energy from solar, battery or the grid. We have taken into consideration a lithium ion battery for this experiment which has a range of $4.4 \mathrm{kWh}$ to $7.5 \mathrm{kWh}$ [6]. These are the high end expensive battery. For the experiment, we assume that the maximum capacity of the battery will be $4.5 \mathrm{kWh}$ and it can charge up to $2.5 \mathrm{kWh}$ in one hour. From [7] and [8], we calculate the amount of power needed by different appliances and their general observed schedule. Based on these numbers, we define allocation vector $\mathrm{x} i$ for each subscriber. We have used a tool to calculate solar energy [10] to get the energy generated by the solar panels. As we can see in Fig.5, there is sufficient amount of power is generated by 15 panels which is sufficient to give electricity to a median home throughout the year. This calculation is done for the Arizona State University location. In our experiment, we have taken readings for the month of January.

\section{A. $\delta$ Calculation}

In Utilitarian algorithm, the value of the delta is set by the energy provider. Energy providers can maximize their profit by changing the value of $\delta$ as discussed in the above sections. During off-peak hours, there is no profit to energy providers, if electricity is given back to the grid. But, in peak hours, when the electricity rates are high along with the demand, user cannot be paid by same electricity price. Hence, energy provider purchase the electricity with the reduced price of $\delta$. Fig. 6 depicts APS charges for subscriber with $\$ 0.20960$ per $\mathrm{kWh}$ during peak hours (12noon-7pm) and $\$ 0.02601$ during off-peak hours [9]. For $\delta=0.01$, APS can buy back the electricity at the price of $\$ 0.02096 \mathrm{kWh}$.

\section{Delta Values}

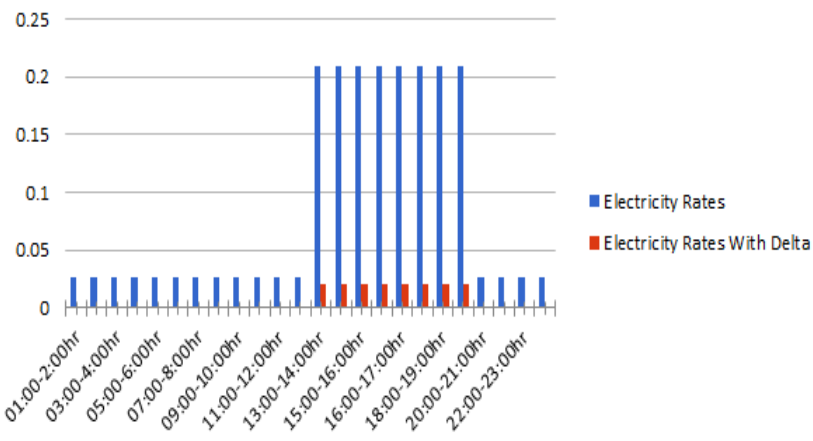

Fig. 5. Delta values.

Energy Map: Use battery in peak and non-peak hours

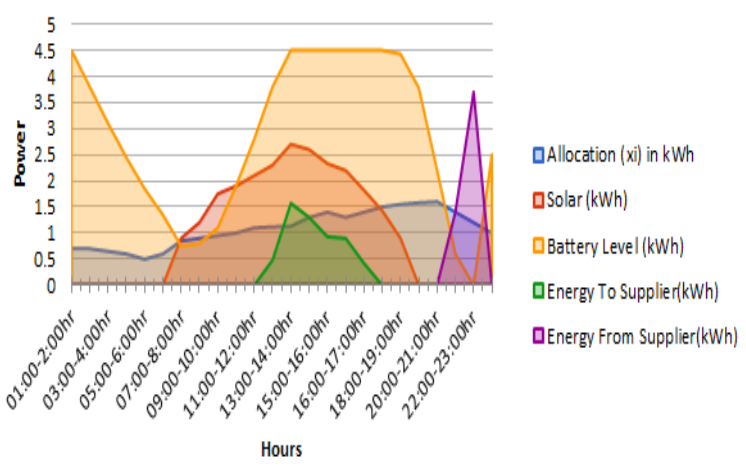

Fig. 6. Energy map with battery in peak and non peak hours. 


\section{1) Total Energy Map}

Till now, we have calculated the allocation vector, solar power generated per hour, battery with finite capacity $(4.5 \mathrm{kWh})$, finite charging rate $(2.5 \mathrm{kWh})$ and $\delta$ factor for peak $(=0.01)$ and off-peak hours $(=0)$. Based on these values, we plot the below graph as seen in Fig. 7 for the whole day.

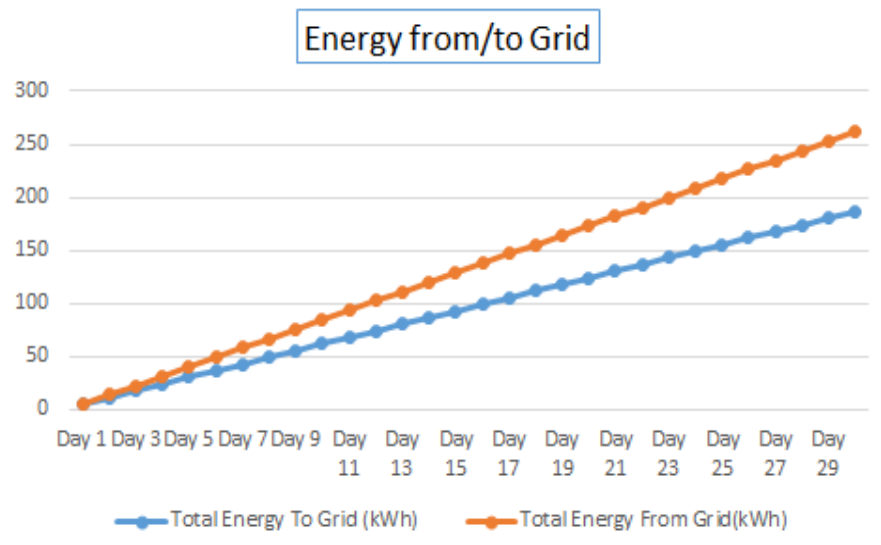

Fig. 7. Subscriber usage of supplier energy.

Initially the battery level is full and hence smart meter based on our algorithm will use battery to fulfil the allocation request. Now, from 6:00 am onwards, the solar energy starts generating the power and can use to fulfil the allocation requests. As the day goes on, more solar energy is generated and this can be used to charge the battery. After 11:00 pm, as the battery is fully charged, the electricity is given back to the power grid. Now, from 4:00 pm onwards, as the sunlight fades, the solar power generation reduces and it can no longer serve the allocations requests. The smart meter now makes a decision to use the power from the battery. By 9:00 pm, the battery is totally drained and hence, smart meter now switches to grid to fulfil the allocation requests. But, as the power is cheap in off-peak hours, smart meter also charges the battery so that the user in next day can send more energy to the grid during peak hours instead of charging the battery.

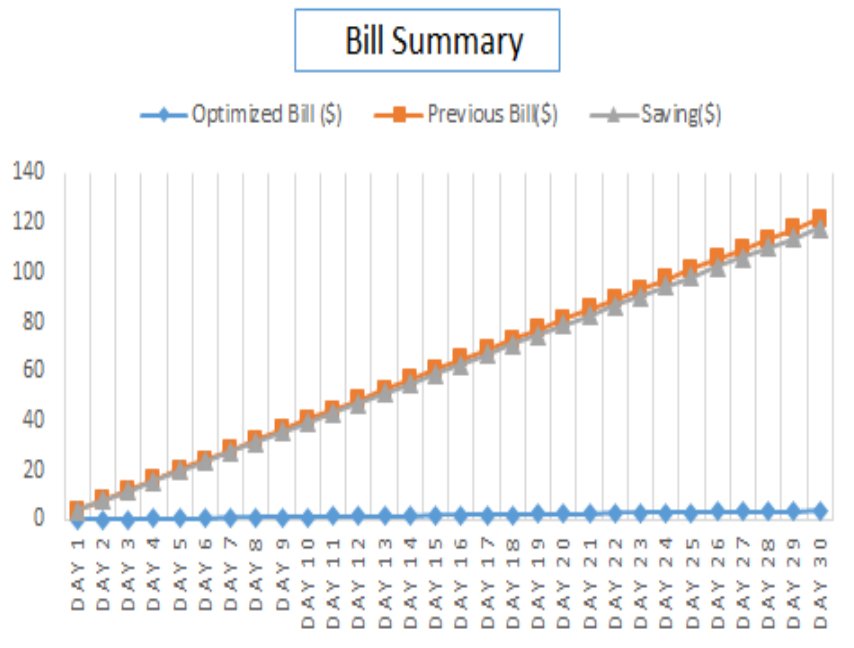

Fig. 8. Saving.

\section{Energy Map: Only use battery in peak hours}

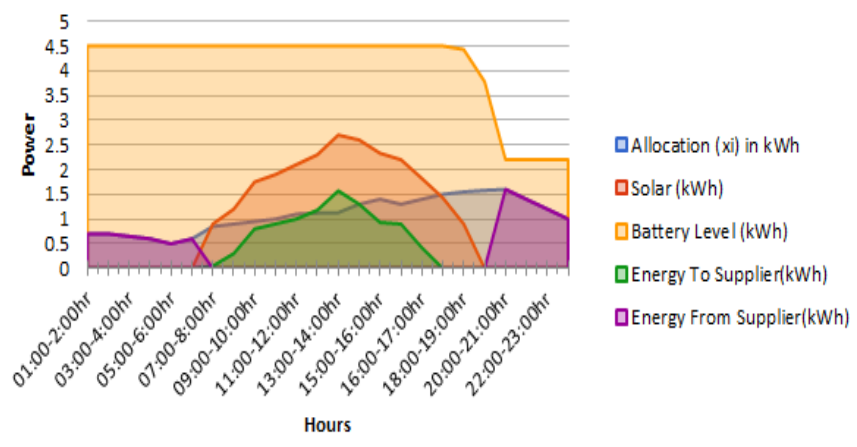

Fig. 9. Energy map: only use battery in peak hours.

After analyzing the whole month usage, we found that the subscriber gives back $186.85 \mathrm{kWh}$ of energy to the grid and took $261.75 \mathrm{kWh}$ of energy from the grid. The subscriber's monthly expected bill was $\$ 121.64469$ before our smart meter addition and it came down to $\$ 3.58$ with total saving of $\$ 118$ in month of January. With this saving, as plotted in Fig. 8, user can buy back the whole solar panel setup cost $(\$ 12 \mathrm{~K})$ [10] in 8 years. The above graph in Fig. 9 shows the Energy Map when the battery is used in peak hours or else power is used from the power grid in non-peak hours. As we can see, more energy is given back to the grid as the battery is charged during non-peak hour period. Here, the battery usage is less, but desirable as it is inexpensive and have limited charging but desirable as it is inexpensive and have limited chargingdischarging cycle.

\section{CONCLUSION}

The re-engineering of Asynchronous Consumption Model [1] is the one of the foundations of our research. We categorize appliances based on the three qualities of service metrics to get a subscriber demand. In this paper, we have formulated a novel way to use demand side management by utilizing multiple energy sources. We have come up with the energy usage algorithm which proves that our approach gives better results to the subscribers based on our analysis. We modelled our system as a Stackelberg game where the energy provider was the leader and the subscribers were the followers. Our pricing model controlled by the provider based on the time slots is another effective strategy which is proven in the results. Overall, the subscriber and the provider utility is maximized which paves way to the maximization of utility of our entire system. We also showed the existence of a Nash equilibrium which is also the optimal case in our design.

A possible future direction would be to dynamically calculate prices for a particular time slot in our pricing scheme.

\section{CONTRIBUTIONS}

Multiple papers have been published in the aspect of demand side management in smart grid. The paper [2] researched on utilizing a storage device during peak hours, but it fails to optimize the subscriber utility in general as it focuses 
only on peak hours. The same issue was in the paper [4] which discusses about storage devices for smart grid. While conducting our research, we took a step back and found the fundamental problem was with energy generation, which is why we focused on solar energy. But since solar energy is inconsistent, we focused on the usage of solar energy to charge a storage device, battery, in our case. Little research [4] in smart grid is in the direction where subscribers can give energy back to the grid. We considered this case and focused our research in this direction and proved that with an effective pricing scheme, subscribers can gain incentives to participate in our plan.

\section{ACKNOWLEDGMENT}

We thank Professor Xue, the teaching assistants, Ruozhou and Xiang for their continued encouragement and support. We have learnt a lot about demand side management and its vast applications in smart grid while independently researching for this paper. To conclude, this course has provided us with solid foundation and knowledge that will definitely help us in our future endeavours.

\section{REFERENCES}

[1] Salfati, E.; Rabinovici, R., "Demand-side management in smart grid using game theory" in Electrical \& Electronics Engineers in Israel (IEEEI), 2014 IEEE 28th Convention of , vol., no., pp.1-5, 3-5 Dec. 2014 doi: 10.1109/EEEI.2014.7005769

[2] Nguyen, Hung Khanh, Ju Bin Song, and Zhu Han. "Demand side management to reduce peak-to-average ratio using game theory in smart grid." Computer Communications Workshops (INFOCOM WKSHPS), 2012 IEEE Conference on. IEEE, 2012.

[3] Saad, Walid et al. 'Game-Theoretic Methods For The Smart Grid: An Overview Of Microgrid Systems, Demand-Side Management, And Smart Grid Communications'. IEEE Signal Process. Mag. 29.5 (2012): 86-105.

[4] Soliman, Hazem M., and Alberto Leon-Garcia. 'Game-Theoretic Demand-Side Management With Storage Devices For The Future Smart Grid'. IEEE Trans. Smart Grid 5.3 (2014): 1475-1485.

[5] Wang, Kun et al. 'A Game Theory-Based Energy Management System Using Price Elasticity For Smart Grids'. IEEE Transactions on Industrial Informatics 11.6 (2015): 1607- 1616.

[6] http://bosch-solar-storage.com/the-battery/lithium-battery/

[7] http://www.energysavings.com/energy-consumption.html

[8] http://www.nrccnrc.gc.ca/eng/dimensions/issue6/smart_technology.html

[9] https://www.aps.com/library/rates/et-2.pdf

[10] http://www.wunderground.com/calculators/solar.html 